\title{
Sudden death in a patient with a third ventricle colloid cyst
}

\author{
Morte súbita em paciente com cisto coloide de terceiro ventrículo \\ Danilo Silva',2, Georgios Matis', Olga Chrysou³, Eduardo V. de Carvalho Junior², Leonardo Costa², Matheus \\ Kitamura², Theodossios Birbilis ${ }^{3}$, Hildo Azevedo Filho²
}

${ }^{1}$ Neurosurgical Department, Weill Cornell Medical College, New York NY, USA;
${ }^{2}$ Neurosurgical Department, Restauração Hospital, Recife PE, Brazil;
${ }^{3}$ Neurosurgical Department, Democritus University of Thrace Medical School, Alexandroupolis, Greece.

Correspondence: Danilo Silva; Weill Cornell Medical College, New York NY, USA; Department of Neurological Surgery; Skull base fellow; 124 west 60 th street Apt 37L; New York NY - USA; E-mail: daniloncr@gmail.com

Conflict of interest: There is no conflict of interest to declare.

Received 21 October 2011; Received in final form 09 November 2011; Accepted 16 November 2011

A 26-year-old female was brought to the Emergency Department for experiencing a sudden and progressive worsening of cephalalgia after sexual intercourse. She eventually became drowsy and not responsive to verbal commands. The patient had a medical history of third ventricle colloid cyst (CC), diagnosed in another institution, and had suffered intermittent headaches for two years.

The neurological examination revealed a patient in profound coma with a Glasgow Coma Scale score (GCS) of 3. Her pupils were bilaterally dilated, with no response to light stimulus. After emergency protocol procedures, the patient was admitted to the Intensive Care Unit (ICU) and a head computed tomography (CT) was obtained. The CT demonstrated hydrocephalus and a hyperdense lesion inside the third ventricle compatible with a previously known third ventricle CC (Fig 1 and 2). Due to the poor neurological status, no treatment was recommended and the patient

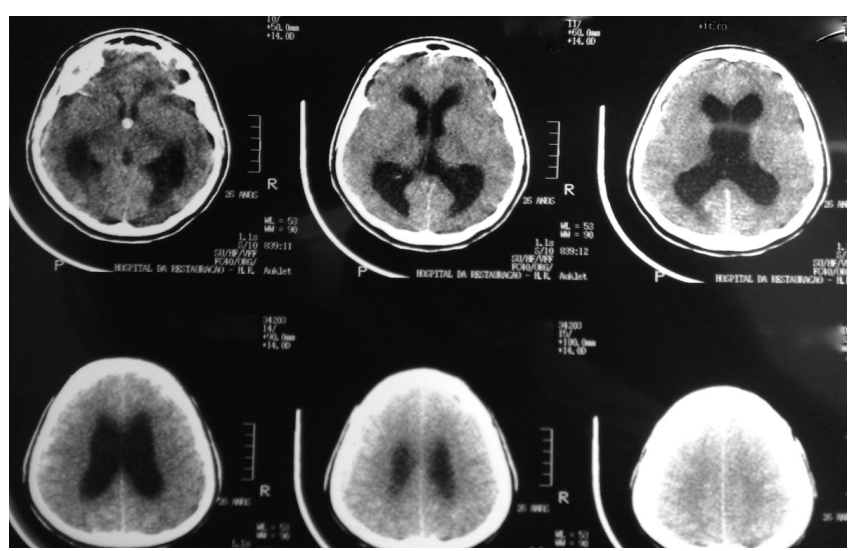

Fig 1. Computed tomography scan demonstrating increased intracranial pressure due to non-communicating hydrocephalus.

\section{References}

died two hours after admission. Institutional ethics committee approval was obtained in order to proceed with this case report.

This is one of the very few cases of sudden death related to a third ventricle CC reported in the literature ${ }^{1-4}$. CCs are regarded as benign lesions ${ }^{1}$, accounting for 0.5 to $2 \%$ of intracranial tumours ${ }^{2}$. The cause of death is believed to be the result of acute cyst swelling due to an intralesional haemorrhage ${ }^{3,4}$. Its incidence varies from 1 to $3 / 10^{6}$ person-years ${ }^{5,6}$. Estimates of the risk of acute deterioration in patients harboring these cysts vary from 6 to $45 \%$ with episodic headache and compromised consciousness, being the most common symptom and sign, respectively ${ }^{5}$. This leads to cerebral herniation as a result of ventricular obstruction after blockage of Monro's foramen ${ }^{1,5,6}$. Since cyst size, duration of symptoms, and presence of hydrocephalus cannot reliably predict the risk of sudden neurological deterioration ${ }^{5}$, surgical management is favored, even when the relationship between the CC and headache is unclear ${ }^{5,6}$.

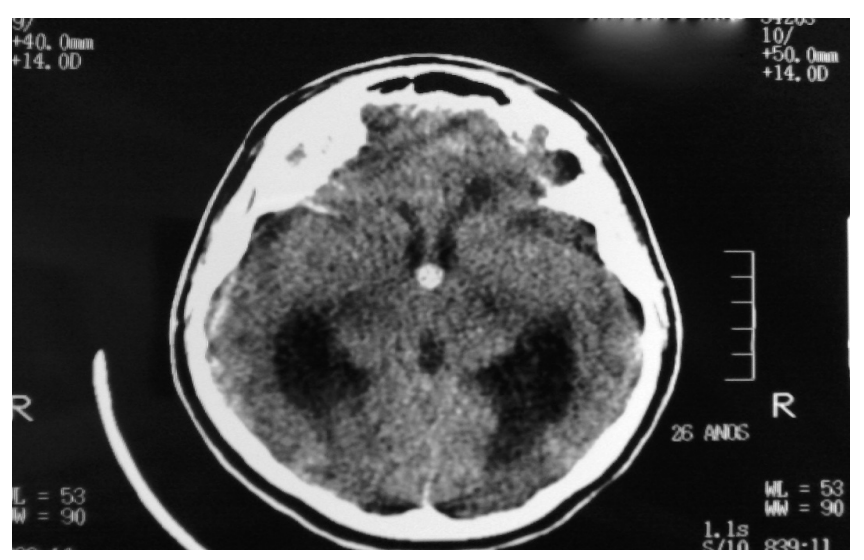

Fig 2. Computed tomography scan illustrating a lesion compatible with a third ventricle colloid cyst.
1. Godano U, Ferrai R, Meleddu V, Bellinzona M. Hemorrhagic colloid cyst with sudden coma. Minim Invasive Neurosurg 2010;53:273-274.

2. Hadar EJ, Schmunk GA, Salamat MS. Hemorrhagic colloid cyst in a patient with leukemia. Case illustration. J Neurosurg 1999;91:516.

3. Malk GM, Horoupian DS, Boulos RS. Hemorrhagic (colloid) cyst of the third ventricle and episodic neurologic deficits. Surg Neurol $1980 ; 13: 73-77$.
4. Beems T, Menovsky T, Lammens M. Hemorrhagic colloid cyst: case report and review of the literature. Surg Neurol 2006;65:84-86.

5. De Witt Hamer PC, Verstegen MJT, de Haan RJ, et al. High risk of acute deterioration in patients harboring symptomatic colloid cysts of the third ventricle. J Neurosurg 2002;96:1041-1045.

6. Pollock BE, Huston 3rd J. Natural History of asymptomatic colloid cysts of the third ventricle. J Neurosurg 1999;91:364-369. 